\title{
Novel method for isoform-centered analysis of alternative splicing using a combination of long and short-read sequencing data
}

\author{
Kasianov A.S. ${ }^{1 *}$, Kovtun A.S. ${ }^{2}$, Penin A.A. ${ }^{1}$ \\ ${ }^{1}$ Institute for Information Transmission Problems RAS, Moscow, Russia \\ ${ }^{2}$ Skolkovo Institute of Science and Technology, Moscow, Russia \\ * email: artem.kasianov@gmail.com
}

Recent advances in DNA sequencing technologies (single molecule sequencing platforms Pacific Biosciences and Oxford Nanopore Technologies) allow direct reading of fragments more than $1 \mathrm{~Kb}$ in length. This paves a way for the analysis of transcriptome complexity focused on isoforms, not on individual splice sites. However, the data obtained by those platforms have high error rate (substitutions, false-positive indels etc.) compared to the $2^{\text {nd }}$ generation. This adversely affects the mapping of the reads on reference genome and leads to the false identification of isoforms. Our preliminary results on the re-analysis of published data on alternative splicing using the long ONT reads show that only $5 \%$ of isoforms are congruent between multiple replicates of the same sample. This problem should be addressed by the development of new algorithms for identification and further analysis of isoforms. We developed a pipeline based on a combination of highly accurate but short reads obtained using Illumina platform and long but error-rich reads from single molecule sequencing. We tested this approach on datasets obtained from cDNA sequencing of Arabidopsis thaliana leaves and anthers on Oxford Nanopore Technologies platform. Samples were taken in two biological replicates; the use of our pipeline yielded the results that were highly congruent between replicates (more than $60 \%$ of isoforms were detected in both replicates). We also applied it to recently published data of direct RNA sequencing (Zhang et al., 2020) and performed a side-by-side comparison of our pipeline and trackcluster software (https://github.com/Runsheng/trackcluster). It showed that our pipeline outperforms trackcluster providing more accurate prediction both known and novel isoforms (approximately $80 \%$ of known isoforms and approximately $60 \%$ of novel isoforms were detected by our pipeline in both replicates versus approximately $75 \%$ of known isoforms and approximately 5\% of novel isoforms were detected by trackcluster in both replicates).

Acknowledgements: The study is supported by Russian Foundation for Basic research (project No. 18-29-13017). 